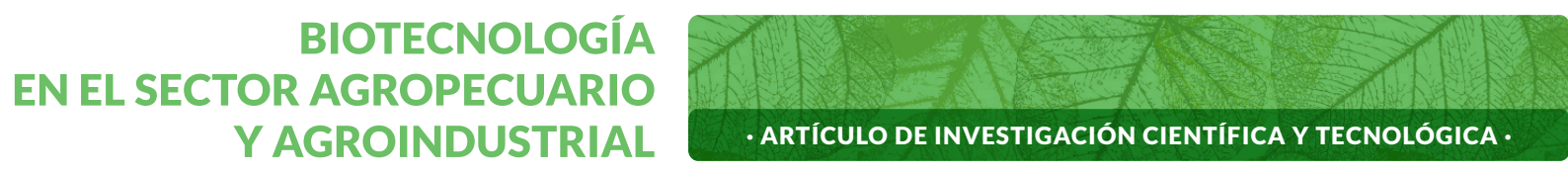

Vol. 19 No 1 · Enero - Junio 2021 · ISSN - 1692-3561 · ISSN-e 1909-9959 · DOI: http://dx.doi.org/10.18684

\title{
Rizobios nativos eficientes en la fijación de nitrógeno en Leucaena leucocephala en Rio Grande do Sul, Brasil*
}

\section{Native rhizobia efficient in nitrogen fixation in Leucaena leucocephala in Rio Grande do Sul, Brazil}

\section{Rizóbios autóctones eficientes na fixação de nitrogênio em Leucaena leucocephala no Rio Grande do Sul, Brasil}

\author{
CUBILLOS-HINOJOSA, JUAN-GUILLERMO ${ }^{1}$; DA SILVA-ARAUJO, FERNANDA²; \\ SACCOL-DE SÁ, ENILSON-LUIZ ${ }^{3}$
}

Historial del Artículo

Recibido para evaluación: 23 de Marzo 2020.

Aprobado para publicación: 20 de Octubre 2020

\footnotetext{
* Titulo del proyecto de origen: "Aplicação conjunta de substâncias húmicas e co-inoculação de rizóbios e Azospirillum brasilense em leguminosas e gramíneas”, Registro SISGEN AEA14C9. Financiación: Universidade Federal do Rio Grande do Sul. Culminación: 26 de junio de 2020.

1 Universidad Popular del Cesar, Departamento de Microbiología. Doctor en Ciencia del Suelo. Valledupar, Colombia. https://orcid.org/0000-0002-3391-420X

2 Universidade Federal do Rio Grande do Sul. Mestranda em Microbiologia Agrícola e do Ambiente. Porto Alegre, Brasil. https://orcid.org/0000-0002-6247-3079

3 Universidade Federal do Rio Grande do Sul, Programa de Pós-graduação em Ciência do Solo, Laboratório de Microbiologia do Solo. Doutor em Ciência do Solo. Porto Alegre, Brasil. https://orcid.org/0000-0002-7796-0857
}

Correspondencia: juancubillos@unicesar.edu.co

Cómo citar este artículo: CUBILLOS-HINOJOSA, JUAN-GUILLERMO; DA SILVA-ARAUJO, FERNANDA; SACCOL-DE SÁ, ENILSON-LUIZ. Rizobios nativos eficientes en la fijación de nitrógeno en Leucaena leucocephala en Rio Grande do Sul, Brasil. Biotecnología en el sector agropecuario y agroindustrial, v. 19, n. 1, 2021, p. xx-xx. Doi: 


\section{RESUMEN}

La selección de rizobios nativos fijadores de nitrógeno (N) es importante para la producción de leucaena utilizada en la producción pecuaria. En el estado de Rio Grande do Sul (RS), Brasil es escasa la información de cepas nativas de rizobios con capacidad simbiótica con plantas de leucaena. El objetivo de este trabajo fue seleccionar rizobios nativos, eficientes en la fijación biológica de $\mathrm{N}$ en plantas de leucaena en RS. Se tomaron muestras de suelo y nódulos en diferentes localidades de RS, luego se utilizaron semillas de leucaena para obtener nódulos que fueron procesados en medios de cultivo para la obtención de los aislamientos. Se realizó un experimento en condiciones de invernadero para evaluar la eficiencia de los rizobios, mediante la determinación de la masa seca de la parte aérea (MSPA), masa seca de la raíz, número de nódulos y $\mathrm{N}$ acumulado de las plantas. Se seleccionaron los aislamientos de rizobios Leu01 y Leu02 como eficientes en la fijación de $N$ por proporcionar aumento de la MSPA y acumulo de $\mathrm{N}$ en las plantas de leucaena similar al tratamiento control que recibió $\mathrm{N}$ mineral. Por tanto, estos aislamientos podrían ser promisores en futuros estudios en invernadero y campo de esta leguminosa.

\section{ABSTRACT}

The selection of native rhizobia nitrogen (N)-fixing is important for the production of leucaena used in livestock production. In the state of Rio Grande do Sul (RS), Brazil there is little information of native isolates of rhizobia with symbiotic capacity with leucaena plants. The objective of this research was to select native rhizobia, efficient in the biological $N$ fixation in leucaena plants in $R S$ state. Soil and nodule samples were taken at different locations in RS, then leucaena seeds were used to obtain nodules that were processed in culture media to obtain the isolates. An experiment under greenhouse conditions was conducted to evaluate the efficiency of rhizobia isolates. The dry shoot dry mass (SDM), dry mass of the root, number of nodules and the $N$ accumulated $\mathrm{N}$ of the plants were determined. The isolates of rhizobia Leu01 and Leu02 were selected as efficient in N fixation because they provide increase in MSPA and an accumulation of $\mathrm{N}$ in leucaena plants similar to the control treatment that received $\mathrm{N}$ mineral. These isolates could be promising in future greenhouse and field studies of this legume.

\section{RESUMO}

A seleção de rizóbios autóctones fixadores de nitrogênio $(N)$ é importante para a produção de leucena utilizada para a produção pecuária. No estado do Rio Grande do Sul (RS), Brasil, existem poucas informações sobre isolados de rizóbios autóctones com capacidade simbiótica com plantas de leucena. O objetivo deste trabalho foi selecionar isolados de rizóbios autóctones, eficientes na fixação biológica de nitrogênio em plantas de leucena no estado do RS. Foram coletadas amostras de solo e nódulos em diferentes locais do RS e, logo foram utilizadas sementes de leucena para obter os nódulos que em seguida foram processados em meios de cultura para obtenção dos isolados. Foi conduzido um experimento em casa de vegetação para avaliar a eficiência dos isolados de rizóbios, determinando-se a massa seca da parte

\section{PALABRAS CLAVE:}

Simbiosis; Leguminosas; Promoción de crecimiento vegetal.

\section{KEYWORDS:}

Symbiosis; Legumes; Plant growth promotion.

\section{PALAVRAS-CHAVE:}

Simbioses; Leguminosas; Promoção de crescimento vegetal. 
aérea, massa seca da raiz, número de nódulos e nitrogênio acumulado nas plantas. Foram selecionados os isolados de rizóbios Leu01 e Leu02 como eficientes na fixação de N por proporcionarem o aumento da MSPA e acumulo de N nas plantas de leucena, semelhante ao tratamento controle não inoculado que recebeu fertilização nitrogenada, sendo estes isolados considerados como promissores que poderiam ser utilizados em outros estudos em casa de vegetação e campo desta leguminosa.

\section{INTRODUCCIÓN}

La producción animal, principalmente la ganadería, es una de las actividades más importante en el estado de Rio Grande do Sul, Brasil, siendo los pastos nativos la base para la alimentación animal. La necesidad de incrementar la producción de alimentos para la producción animal ha permitido el estudio de leguminosas como Leucaena leucocephala por su versatilidad, siendo utilizada como forraje, asociada con pastos y otros cultivos anuales, el desarrollo de sistemas silvopastoriles y agroforestales, el control de maleza por alelopatía, la recuperación de áreas degradadas y reforestación en suelos tropicales [1,2,3,4,5,6,7].

En el Estado de Rio Grande do Sul, debido a las condiciones subtropicales como las bajas temperaturas en el invierno y la acidez de los suelos, se han realizado estudios sobre el crecimiento y supervivencia de especies y accesiones de leucaena, dentro de las cuales se encuentra Leucaena leucocephala; determinándose una tasa alta de crecimiento y sobrevivencia a las condiciones edafoclimáticas del sur de Brasil, siendo reportada como una de las especies de mayor potencial para ser cultivada [8].

Por otra parte, algunos estudios demuestran que L. leucocephala posee la capacidad de establecer asociación simbiótica con diversos géneros de rizobios como Rhizobium, Bradyrhizobium, Sinorhizobium y Mesorhizobium con capacidad de fijar $\mathrm{N}[3,9,10,11]$ a través de la formación de estructuras conocidas como nódulos que facilitan la captación de $\mathrm{N}$ atmosférico por medio de la fijación biológica de $\mathrm{N}$.

En Brasil, existen cepas de rizobios que se encuentran en la colección SEMIA del Departamento de Diagnóstico e Investigación Agropecuaria (DDPA), recomendadas para leucaena y liberadas para la producción de inoculantes por el Ministerio de Agricultura, Pesca y Abastecimiento (MAPA) de Brasil. Sin embargo, en el estado de Rio Grande do Sul no se han desarrollado estudios de aislamiento y selección de rizobios nativos, adaptadas a las condiciones subtropicales y capaces de establecer asociación simbiótica con leucaena.

Por lo tanto, el objetivo de esta investigación fue seleccionar rizobios nativos en el estado de Rio Grande do Sul simbiontes de leucaena, eficientes en la fijación biológica de nitrógeno que puedan ser utilizados como inoculantes en los sistemas de producción agropecuaria.

\section{MÉTODO}

Este estudio se realizó en el Laboratorio de Microbiología de Suelos y en el invernadero del Departamento de Suelos de la Universidad Federal do Rio Grande do Sul (UFRGS). Se tomaron muestras de suelo en diferentes lugares de las zonas rurales del estado de Rio Grande do Sul, Brasil (Cuadro 1). El muestreo se realizó a juicio a una profundidad de 0 a $20 \mathrm{~cm}$, considerándose la presencia de plantas leguminosas nativas o introducidas. Adicionalmente, fueron recolectados nódulos de plantas de Desmodium sp en la Estación Experimental Agronómica (EEA) de la UFRGS en el municipio Eldorado do Sul.

\section{Aislamiento y caracterización de rizobios}

Las muestras de suelo fueron recolectadas y transportadas al Laboratorio de Microbiología del Suelo y posteriormente se colocaron $10 \mathrm{~g}$ de cada muestra en $90 \mathrm{~mL}$ de solución salina al $0,85 \%$, manteniéndose en agitación durante tres horas. 
Cuadro 1. Localización de las muestras recolectadas.

\begin{tabular}{|c|c|c|c|c|}
\hline Muestra & Lugar & Latitud (S) & Longitud (O) & Leguminosa predominante en el área \\
\hline 1 & Eldorado do Sul - RS & $30^{\circ} 5^{\prime} 19.03^{\prime \prime}$ & $51^{\circ} 40^{\prime} 28.21^{\prime \prime}$ & Desmodium sp. \\
\hline 2 & Eldorado do Sul - RS & $30^{\circ} 5^{\prime} 16.75^{\prime \prime}$ & $51^{\circ} 40^{\prime} 29.24^{\prime \prime}$ & Desmodium sp. \\
\hline 3 & Eldorado do Sul - RS & $30^{\circ} 6^{\prime} 58.50^{\prime \prime}$ & $51^{\circ} 39^{\prime} 57.98^{\prime \prime}$ & Bracatinga (Mimosa sp) \\
\hline 4 & Porto Alegre - RS & $30^{\circ} 4^{\prime} 15.87^{\prime \prime}$ & $51^{\circ} 8^{\prime} 20.55^{\prime \prime}$ & Leucaena (Leucaena leucocephala) \\
\hline 5 & Viamão - RS & $30^{\circ} 7^{\prime} 43.57^{\prime \prime}$ & $51^{\circ} 4^{\prime} 4.44^{\prime \prime}$ & Maricá (Mimosa bimucronata) \\
\hline 6 & Viamão - RS & $30^{\circ} 7^{\prime} 51.18^{\prime \prime}$ & $51^{\circ} 3^{\prime} 58.67^{\prime \prime}$ & Inga (Inga marginta) \\
\hline 7 & Viamão - RS & $30^{\circ} 7^{\prime} 50.46^{\prime \prime}$ & $51^{\circ} 4^{\prime} 0.46^{\prime \prime}$ & Arbórea sem identificar \\
\hline
\end{tabular}

Para el aislamiento de rizobios a partir del extracto de suelo se instaló un experimento, utilizando como trampa semillas de plantas de leucaena recolectadas en campo para obtener nódulos. Estas semillas fueron escarificadas con papel de lija No. 100 durante 1 minuto y posteriormente desinfectadas por inmersión sucesiva en alcohol al 70\% durante 30 segundos, hipoclorito de sodio al 2,5\% durante 30 segundos e inmediatamente se enjugaron seis veces consecutivas con agua destilada estéril [12]. Luego, las semillas se sembraron por separado en vasos plásticos que contenían una mezcla de vermiculita y arena (2:1) e inmediatamente se realizó la adición de $2 \mathrm{~mL}$ de la suspensión liquida de las muestras de suelo en cada vaso, utilizándose 5 repeticiones para cada muestra. Los vasos fueron regados con la solución nutritiva Sarruge [13], para mantener a las plántulas durante el experimento en condiciones de laboratorio.

Transcurridos 45 días después de adición de la suspensión de suelo, se realizó la separación de los nódulos de las raíces de las plántulas de leucaena y posteriormente se desinfectaron, utilizándose la misma metodología descrita anteriormente [12]. Posteriormente, en cabina de flujo laminar los nódulos fueron macerados por separado con un bastón de vidrio en tubos de ensayo estériles. La suspensión obtenida de los nódulos macerados se inoculó en placas de Petri con medio de cultivo de levadura manitol con rojo congo (LMR) en cabina de flujo laminar utilizando el método de la gota y dispersión por estrías [14]. Seguidamente, las placas fueron incubadas a $28^{\circ} \mathrm{C} \pm 2$ durante 10 días. Los nódulos recogidos en campo de Desmodium sp. fueron desinfectados y procesados de la misma manera que fue descrita anteriormente.

Todos los aislamientos bacterianos fueron transferidos a otras cajas de Petri con medio LMR con el propósito de obtener colonias con morfología persistente. Se realizó una evaluación diaria del crecimiento bacteriano de cada placa y con la ayuda de un microscopio estereoscópico, se determinó la morfología colonial, observándose el diámetro y color de la colonia, tipo de borde, opacidad, consistencia y tiempo de crecimiento [15]. Además, se evaluó el cambio de $\mathrm{pH}$ en el medio de cultivo de levadura manitol con adición de azul de bromotimol (LMA+AB). Los aislamientos puros se mantuvieron en tubos de ensayo de medio LMA en refrigeración a $4^{\circ} \mathrm{C}$.

\section{Evaluación de la capacidad simbiótica in vitro de los aislamientos obtenidos}

Los aislamientos obtenidos a partir de nódulos generados por la adición de la suspensión de las muestras de suelos en plantas de leucaena y los aislamientos obtenidos a partir de nódulos de Desmodium sp. recolectados en campo, además de tres cepas de rizobios Vp16, Lc348, Din3 del banco de cepas de rizobios del Laboratorio de Microbiología de Suelos de la UFRGS, fueron evaluados en cuanto a su capacidad para inducir la formación de nódulos en plantas de leucaena en condiciones in vitro. Para esto, se usaron tubos de ensayo que contenían 30 $\mathrm{mL}$ de solución nutritiva de Sarruge [13] al 25\% semisólida, previamente esterilizado en autoclave.

Los aislamientos obtenidos fueron transferidos para obtener los inóculos en tubos falcon que contenían $30 \mathrm{~mL}$ de medio de cultivo líquido de levadura manitol (LM) y se mantuvieron en una incubadora orbital a $28 \pm 2^{\circ} \mathrm{C}$ con agitación a 120 rpm según el tiempo de crecimiento de cada aislamiento, hasta alcanzar una concentración de $10^{8}$ células. $\mathrm{mL}^{-1}$ verificada por medio de recuento en placa. 
En cabina de flujo, en cada tubo fue colocada una semilla de leucaena pre-germinada en cabina de flujo laminar, realizándose 3 repeticiones por cada aislamiento bacteriano. Luego, se inoculó $1 \mathrm{~mL}$ del caldo de cada aislamiento en cada tubo. Después de la inoculación, los tubos se mantuvieron en un lampadario con $8 \mathrm{~h}$ luz por día a temperatura ambiente de $30^{\circ} \mathrm{C}$ aproximadamente. Se utilizó como control un tubo no inoculado. Transcurrido un período de 40 días, fue observada la formación de nódulos en las raíces de las plantas y verificado si estos se encontraban activos por la coloración rojiza que genera la actividad de la leg-hemoglobina, que indica la fijación biológica de $\mathrm{N}$.

\section{Evaluación de la eficiencia simbiótica en la fijación de nitrógeno por los rizobios}

Los aislamientos de rizobios fueron seleccionados del experimento in vitro anteriormente descrito, por su capacidad de inducir la formación de nódulos en plantas de leucaena. Estos aislamientos, fueron evaluados en cuanto a su eficiencia en la fijación biológica de $\mathrm{N}$ en condiciones de invernadero. Para esto, cinco semillas de leucaena previamente escarificadas y desinfectadas fueron sembradas en vasos Leonard, que contenían una mezcla de vermiculita y arena (2:1) en la superior y en la parte inferior solución nutritiva Sarrugue [13] exenta de N. Seguidamente, se realizó la inoculación de las semillas con los aislamientos de rizobios seleccionados, adicionando 2 $\mathrm{mL}$ de inóculo en concentración de $10^{-8}$ células. $\mathrm{mL}^{-1}$. Se utilizaron como tratamiento control las cepas de la colección SEMIA: 6361 (Bradyrhizobium sp), 6097 (Bradyrhizobium sp), 4077 (Rhizobium tropici CIAT 899) recomendadas para leucaena y liberadas por el Ministerio Agricultura, Pecuaria y Abastecimiento (MAPA) de Brasil para la industria de inoculantes. Una semana después, se hizo el raleo de las plantas dejando solo una planta por vaso.

Se siguió un diseño experimental de bloques al azar con 17 tratamientos y cinco repeticiones, donde fueron evaluados 11 aislamientos, 2 cepas recomendadas de la colección de cepas "SEMIA" de rizobios y dos controles sin inoculación. Uno de estos controles sin inocular fue sin adición de $\mathrm{N}$ (Control-N) y el otro con adición de $\mathrm{N}$ (Control+N), utilizándose una dosis de $\mathrm{N}$ de $100 \mathrm{~kg} \cdot \mathrm{ha}{ }^{-1}$ en la forma de nitrato de amonio $\left(\mathrm{NH}_{4} \mathrm{NO}_{3}\right)$. Esta dosis

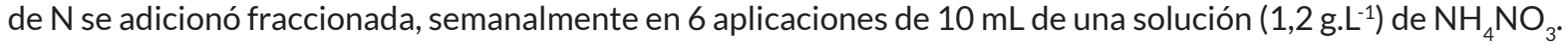

Transcurrido un periodo de 60 días de cultivo, las plantas fueron retiradas de los vasos, separando la parte aérea del sistema radicular. Se determinó la masa seca de la parte aérea (MSPA), la masa seca de la raíz (MSR), el número de nódulos (NN) y el nitrógeno total acumulado (Nac) de la parte área y posteriormente el índice de eficiencia relativa (IER) [16] de la fijación biológica de $\mathrm{N}$ de los aislamientos de rizobios. Para esto, la parte aérea de las plantas se colocó en bolsas de papel y se dejó secando en un horno de circulación forzada a $65^{\circ} \mathrm{C}$ durante tres días, luego se procedió a molerlas para la posterior determinación de N [17]. Las raíces fueron lavadas con el fin de eliminar las partículas del sustrato adheridas, los nódulos se separaron para su cuantificación y las raíces se secaron en el horno y se pesaron en la balanza.

El IER se calculó utilizando la fórmula (Ec.1) [16]:

$$
E F R=((N T-N T-N) /(N T+N-N T-N)) \times 100
$$

Donde:

NT = nitrógeno total de la planta con tratamiento inoculado.

NT-N = nitrógeno total del tratamiento control no inoculado y sin adición de nitrógeno, que en este caso fue considerado como $0 \mathrm{mg}$ debido a que la masa seca de este tratamiento fue poca, lo que no permitió su análisis. Sin embargo, las plantas tienen una baja cantidad de nitrógeno que proviene de la reserva de la semilla [18].

$\mathrm{NT}+\mathrm{N}$ = nitrógeno total del tratamiento control no inoculado con adición de nitrógeno. 
Los datos obtenidos se sometieron a análisis de varianza y a la prueba de comparación de medias de Tukey $(p<0,05)$, utilizando el paquete estadístico SPSS 15.

\section{RESULTADOS}

\section{Aislamientos obtenidos a partir de muestras de suelo}

En el aislamiento a partir de muestras de suelo utilizando plantas de leucaena como trampa para obtener nódulos, se obtuvieron 17 aislamientos, distribuidos así: 2 a partir de la muestra 1 (Leu09, Leu10), 2 de la muestra 2 (Leu11, Leu12), 1 de la muestra 3 (Leu13), 8 de la muestra de 4 (Leu01, Leu02, Leu03, Leu04, Leu05, Leu06, Leu07, Leu08), 1 a partir de la muestra 5 (Leu14), 1 de la muestra 6 (Leu15) y 2 de la muestra 7 (Leu16, Leu17) (Cuadro 2). Resultados similares fueron encontrados en un estudio de caracterización de rizobios simbiontes de leucaena en diferentes locales en Mérida (México), donde se obtuvieron 23 aislamientos de rizobios [15].

Se observó una escasa nodulación a partir de las muestras de suelo de algunos puntos de muestreo y esto puede deberse a que no existían cultivos o plantas de leucaena en estas localidades, con excepción de la muestra 4 donde se obtuvo el mayor número de aislamientos con 47 \% (Figura 1) que fue tomada de suelo rizósferico de una planta de leucaena. Las plantas de leucaena se caracterizan por presentar una alta especificidad para establecer asociación simbiótica con rizobios, lo que limita la fijación biológica de $\mathrm{N}$ con los rizobios que forman nódulos $[11,20]$. En un estudio de aislamientos de rizobios en diferentes puntos de muestreo en campo, fueron encontrados también una baja nodulación, atribuyendo esto a la inexistencia de cultivos de leucaena [20].

A partir de los nódulos de plantas de Desmodium sp recolectadas en el campo se obtuvieron 12 aislamientos, que posteriormente fue determinado si tenían la capacidad de formar nódulos en las plantas de leucaena (Cuadro 2).

\section{Evaluación de la capacidad simbiótica in vitro de los aislamientos obtenidos}

En la prueba de evaluación de la capacidad simbiótica in vitro de los aislamientos obtenidos, fue evidenciada por la producción de la coloración rojiza al interior del nódulo por actividad de la leg-hemoglobina, siendo determinados como rizobios simbiontes de leucaena los aislamientos Leu01, Leu02, Des04, Des05, Des06, Des09, Des15 y Des23 (Cuadro 2), los cuales fueron seleccionados para el experimento de eficiencia en la fijación biológica de nitrógeno. Las tres cepas Vp16, Lc348, Din3 del banco de aislamientos de rizobios del Laboratorio de Microbiología de Suelos de la UFRGS, que fueron incluidas en este experimento no tuvieron la capacidad para inducir la formación de nódulos, a pesar de su capacidad de establecer simbiosis con otras leguminosas [12].

La evaluación de simbiosis de los aislamientos con leucaena fue determinada por la actividad de la leghemoglobina evidenciada nos nódulos siendo considerados fix (+) activos, fix (-) negativo. Des: Desmodium sp.; Leu: Leucaena leucocephala; LMA+AB: Levedura manitol agar+ azul de bromotimol

\section{Evaluación de la eficiencia en la fijación biológica de nitrógeno de los rizobios}

Los resultados de MSPA, MSR, NN, Nac y el índice IER determinados en las plantas de leucaena inoculadas con los aislamientos y las cepas SEMIA de rizobios son presentados en el Cuadro 3.

Se encontró una mayor masa seca en los tratamientos con los aislamientos de rizobios Leu01, Leu02, las tres cepas SEMIA 6361, SEMIA 6097 y SEMIA 4077, presentando diferencias con el tratamiento control no inoculado y sin adicion de $\mathrm{N}$ (Control-N), como tampoco hubo diferencias con el tratamiento control no inoculado, que recibió adición de nitrógeno (Control+N) según la prueba de Tukey. Estos resultados indican que tanto los aislamientos de rizobios Leu01 y Leu02 como las cepas SEMIA promueven el crecimiento de las plantas de leucaena. Resultados similares fueron obtenidos en un estudio con cepas nativas de Rhizobium sp inoculadas en plantas de 


\begin{tabular}{|c|c|c|c|c|c|c|c|c|c|c|c|c|c|c|c|c|c|c|}
\hline 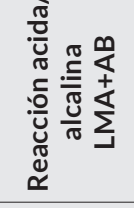 & 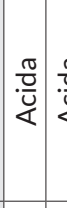 & $\frac{\pi}{\tilde{T}}$ & 弯 & 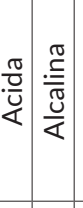 & \begin{tabular}{l|l}
$\frac{\pi}{0}$ \\
$\frac{\pi}{4}$ & $\frac{8}{4}$ \\
\end{tabular} & & 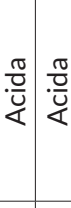 & $\frac{\pi}{\frac{\pi}{4}} \frac{\pi}{\frac{\pi}{4}}$ & $\frac{\frac{\pi}{0}}{\frac{0}{4}} \frac{\frac{\pi}{6}}{\frac{0}{4}}:$ & 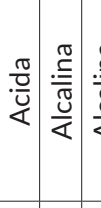 & $\begin{array}{l}\frac{\pi}{\frac{5}{5}} \\
\frac{0}{4} \\
\frac{\pi}{4} \\
\frac{\pi}{4}\end{array}$ & 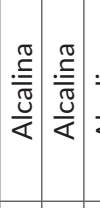 & 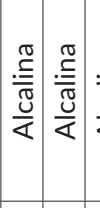 & 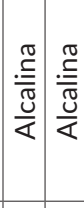 & 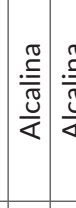 & 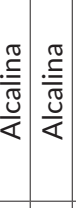 & & 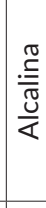 \\
\hline 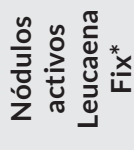 & & ++ & + & $1+$ & $1+$ & & + & & ++ & & .1 & . & & & ' & & & ' \\
\hline 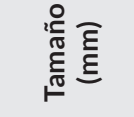 & in 7 & $r$ & in & $\begin{array}{lll}0 & \text { in }\end{array}$ & $n$ & & & & $\nabla \infty$ & $N \rightarrow \sigma^{\circ}$ & $m \sim c$ & $m \rightarrow$ & $A$ & $\begin{array}{lll}\overrightarrow{v_{1}} & \stackrel{\sim}{\sim} \\
\rightarrow\end{array}$ & $\vec{v}$ & $\overrightarrow{v_{1}} \mid-1$ & & - \\
\hline 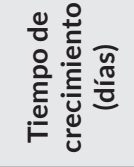 & $\diamond$ & $\checkmark m$ & $\nabla$ & $m+$ & ১ & & $\diamond \triangleleft$ & ১ & $m$ & $m$ in & $m$ m & m & $\nabla$ in & in & in is & n $n$ & & in \\
\hline 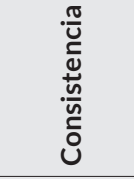 & 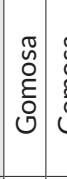 & 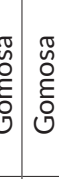 & 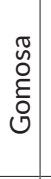 & 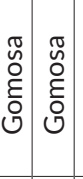 & 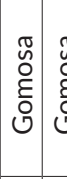 & & & 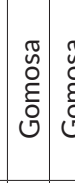 & 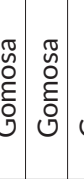 & 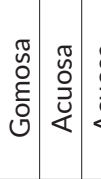 & 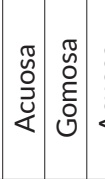 & 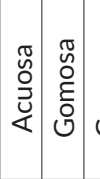 & 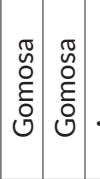 & 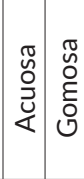 & 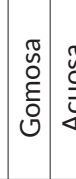 & 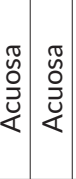 & & 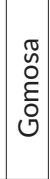 \\
\hline 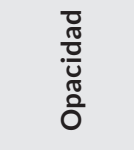 & 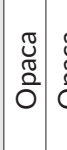 & 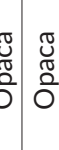 & 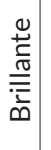 & 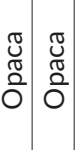 & 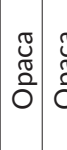 & 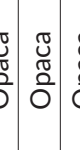 & 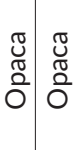 & & 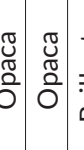 & 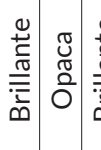 & 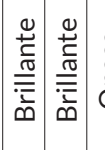 & 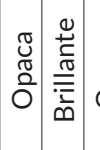 & 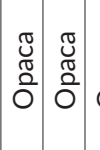 & 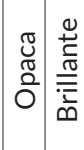 & 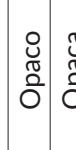 & 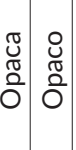 & & 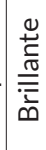 \\
\hline$\frac{\grave{0}}{\mathrm{O}}$ & 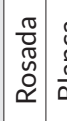 & 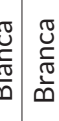 & $\begin{array}{l}\frac{\pi}{\tilde{T}} \\
\frac{\pi}{\omega}\end{array}$ & 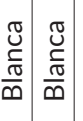 & 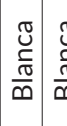 & & 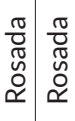 & 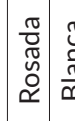 & 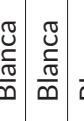 & 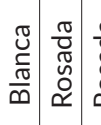 & 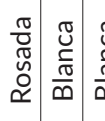 & 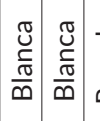 & 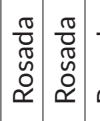 & 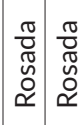 & 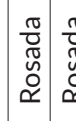 & & & $\begin{array}{l}0 \\
0 \\
0 \\
0 \\
0 \\
\alpha\end{array}$ \\
\hline 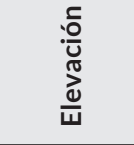 & & & & & & & & & & 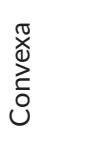 & & & & & & & & \\
\hline 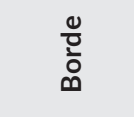 & & & & & & & & & & 迹 & & & & & & & & \\
\hline$\stackrel{\widetilde{\sigma}}{\tilde{5}}$ & & & & & 竞 & & & & & 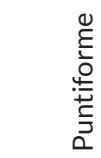 & E. & & 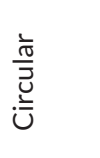 & & 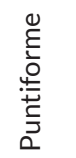 & & 克 & \\
\hline 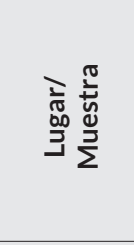 & & & $\begin{array}{l}5 \\
6 \\
5 \\
5 \\
5 \\
5 \\
\\
\\
\end{array}$ & & $\begin{array}{l}N \\
\bar{J} \\
0 \\
0 \\
\frac{0}{0} \\
\frac{0}{0} \\
\frac{0}{0}\end{array}$ & & $\begin{array}{l}\stackrel{m}{5} \\
\overline{5} \\
\frac{0}{0} \\
\frac{0}{0} \\
\frac{0}{0} \\
\frac{0}{4}\end{array}$ & & & $\begin{array}{l}\frac{1}{0} \\
\frac{0}{\delta 0} \\
\frac{d}{<} \\
0 \\
\frac{0}{0} \\
\frac{0}{0}\end{array}$ & 管 & & $\begin{array}{l}\frac{1}{\overline{5}} \\
\overline{0} \\
\frac{0}{0} \\
\frac{0}{0} \\
\frac{0}{0} \\
\frac{0}{4}\end{array}$ & 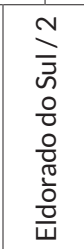 & 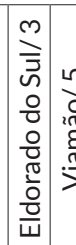 & 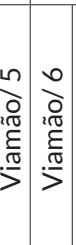 & & 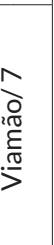 \\
\hline 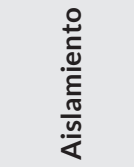 & 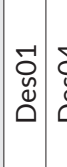 & ธे & & $\infty a_{0}$ & 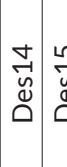 & & 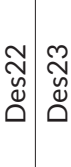 & $\check{c}$ & & 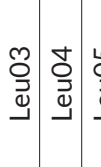 & 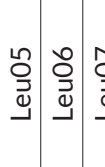 & 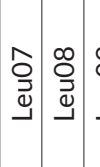 & : & ت: & 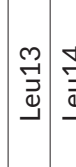 & ت্ড: & : & $\stackrel{亏}{\Xi}$ \\
\hline
\end{tabular}


leucaena, donde las cepas contribuyeron en el aumento la masa seca de las plantas [11]. En contraste, difieren de los resultados encontrados en un estudio realizado con cepas de la colección de rizobios del Instituto Nacional de Investigaciones de la Amazonia (INPA) de Brasil, que fueron inoculadas en plantulas de leucaena, evaluando el efecto sobre la masa seca y comparadas con el control sin inocular y que recibió fertilizacion nitrogenada, no encontrando diferencias entre los tratamientos [21].

Por otra parte, fue evidenciado en las plantas de leucaena inoculadas con los aislamientos de rizobios obtenidos de nodulos de Desmodium sp. una baja MSPA, semejante al tratamiento control no inoculado sin adicion de $\mathrm{N}$ (Controle-N). Estos resultados pueden estar asociado a la baja nodulación presentada, a pesar de que estos aislamientos de rizobios fueron determinados como simbiontes de leucaena en la prueba de capacidad simbiótica,

Figura 1. Porcentaje de aislamientos obtenidos de muestras de suelo por localidades del Estado de Rio Grande do Sul, Brasil.

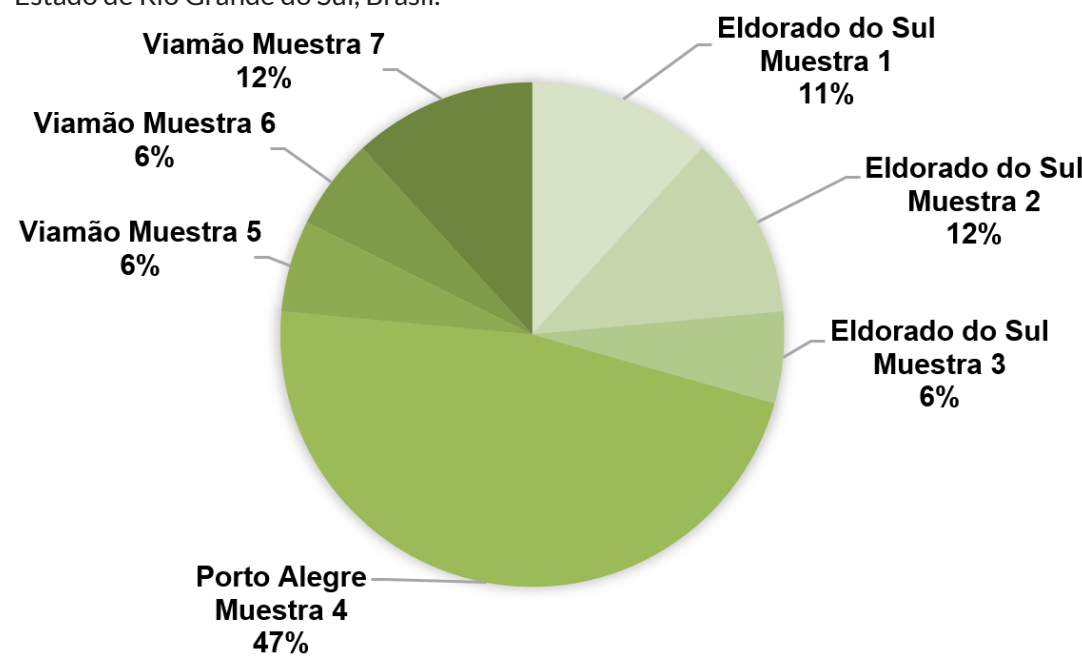

Cuadro 3. Valores promedio de masa seca de la parte aérea (MSPA), masa seca de la raíz (MSR), número de nódulos (NN), nitrógeno acumulado (Nac) de las plantas inoculadas con rizobios y el índice de eficiencia relativa (IER).

\begin{tabular}{|c|c|c|c|c|c|}
\hline \multirow{2}{*}{ Tratamientos } & MSPA & MSR & \multirow{2}{*}{$\begin{array}{c}\text { NN } \\
-\end{array}$} & \multirow{2}{*}{$\begin{array}{c}\mathrm{Nac} \\
\mathrm{mg}\end{array}$} & \multirow{2}{*}{$\begin{array}{c}\text { IER } \\
\% \\
\end{array}$} \\
\hline & \multicolumn{2}{|c|}{ g } & & & \\
\hline Control+N & $0,35 a$ & $0,24 \mathrm{a}$ & $\mathrm{Od}$ & $6,32 \mathrm{a}$ & 100 \\
\hline SEMIA 6361 & $0,41 a$ & $0,24 a$ & $30 \mathrm{~b}$ & $10,78 \mathrm{a}$ & 141 \\
\hline SEMIA 6097 & $0,33 a$ & $0,24 a$ & $32 b$ & $7,69 a$ & 113 \\
\hline SEMIA 4077 & $0,43 a$ & $0,20 \mathrm{a}$ & $35 a b$ & $10,83 a$ & 148 \\
\hline Leu01 & $0,37 a$ & $0,21 a$ & $40 a$ & $9,31 \mathrm{a}$ & 129 \\
\hline Leu02 & $0,37 a$ & $0,21 \mathrm{a}$ & $34 b$ & $8,00 \mathrm{a}$ & 127 \\
\hline Des04 & $0,14 b$ & $0,09 a$ & $6 c$ & $0,00^{*}$ & 0 \\
\hline Des05 & $0,14 b$ & $0,11 a$ & $8 c$ & $0,00^{*}$ & 0 \\
\hline Des06 & $0,16 b$ & $0,11 \mathrm{a}$ & $8 c$ & $0,00^{*}$ & 0 \\
\hline Des09 & $0,12 \mathrm{~b}$ & $0,13 a$ & $9 c$ & $0,00^{*}$ & 0 \\
\hline Des15 & $0,15 b$ & $0,10 a$ & $9 c$ & $0,00^{*}$ & 0 \\
\hline Des23 & $0,16 b$ & $0,12 \mathrm{a}$ & $9 c$ & $0,00^{*}$ & 0 \\
\hline Control-N & $0,10 b$ & $0,09 a$ & $0 \mathrm{~d}$ & $0,00^{*}$ & 0 \\
\hline
\end{tabular}

* No determinado en este tratamiento por la poca cantidad de muestra. Letras distintas indican diferencias significativas entre tratamientos, según prueba de tukey al $5 \%$. 
concordando con un trabajo similar [20], donde fue reportado que no todos los rizobios que establecen simbiosis con leguminosas son eficientes en la fijacion biologica de $\mathrm{N}$.

En la variable masa seca de la raíz, no fueron observadas diferencias entre los tratamientos, lo que indica que los rizobios no estimularon el crecimiento radicular de plantas de leucaena.

En relación al número de nódulos (NN) se evidenció que los aislamientos de rizobios Leu01, Leu02 y las tres cepas (SEMIA 6361, SEMIA 6097 y SEMIA 4077) fueron los que tuvieron mayor capacidad de formar nódulos en comparación con los aislamientos de nódulos de Desmodium sp. simbiontes de leucaena que presentaron una baja nodulación. Estos resultados coinciden con los observados en un estudio de establecimiento de sistemas silvopastoriles en campo, donde semillas de leucaena fueron inoculadas con el producto comercial Ferbiol que contenía la cepa Rhizobium loti, cultivadas en vivero y posteriormente sembradas en campo, durante ocho semanas, donde no evidenciaron diferencias en el número de nódulos entre las plantas inoculadas y no inoculadas, sin embargo encontraron que los nódulos de las plantas no inoculadas se encontraban inactivos, ya que son nódulos establecidos por rizobios autóctonos del suelo [3]. Otros resultados similares fueron evidenciados en una investigación realizada en invernadero con aislamientos Rhizobium sp. nativos y una cepa comercial inoculados en semillas y plántulas de leucaena, que estimularon la nodulación de las plantas favoreciendo la fijación de N [11].

En el $\mathrm{N}$ acumulado por las plantas, los resultados obtenidos muestran que los aislamientos de rizobios Leu01, Leu02 y las tres cepas (SEMIA 6361, SEMIA 6097 y SEMIA 4077) proporcionaron una acumulación de N similar al tratamiento control no inoculado que recibió fertilización nitrogenada (Controle+N) según la prueba de Tukey, con la diferencia de que nitrógeno acumulado por las plantas inoculadas con rizobios es proveniente de la fijación biológica de $\mathrm{N}$ y de la reserva que poseen las semillas [18]. Resultados similares fueron encontrados en un estudio, donde los aislamientos de Rhizobium sp. y las cepas comerciales incrementaron el porcentaje de nitrógeno en las plantas de leucaena inoculadas favoreciendo el crecimiento vegetal en condiciones de invernadero [11].

Por otra parte, el índice de eficiencia relativa (IER) (BROCKWELL et al., 1966) que muestra el porcentaje de eficiencia de una bacteria en asociación simbiótica con leguminosas en fijar N, permitió evidenciar que tanto los aislamientos Leu01 y Leu02 como las tres cepas (SEMIA 6361, SEMIA 6097 y SEMIA 4077) presentaron porcentaje de eficiencia similar o superior al control no inoculado, que recibió fertilización nitrogenada (Control $+\mathrm{N}$ ), lo que permite inferir que estos aislamientos y cepas son eficientes en la fijación biológica de $\mathrm{N}$.

\section{CONCLUSIONES}

Los aislamientos de rizobios Leu01 y Leu02 obtenidos en este estudio son eficientes en fijar nitrógeno en asociación simbiótica con plantas de leucaena. Por tanto, podrían ser utilizados en próximas etapas de estudios de invernadero y campo, para su evaluación agronómica que comprueben su potencial como futuras cepas de inoculantes.

Los aislamientos de rizobios obtenidos de nódulos de Desmodium sp. no son eficientes en la fijación biológica de nitrógeno en plantas de leucaena.

\section{REFERENCIAS}

[1] JETANA, THONGSUK. Potential Benefits from the Utilization of Leucaena leucocephala in Thai Swamp Buffaloes Production. Proceedings of International Seminar on Livestock Production and Veterinary Technology, 2017, p. 90-103.

10.14334/Proc.Intsem.LPVT-2016-p.90-103 
[2] NICODEMO, M.; GARCIA, A.; PORFIRIO-DA SILVA, V.; PACIULLO, D. Desempenho, saúde e conforto animal em sistemas silvipastoris no Brasil. Brasilia D.F. (Brasil): Embrapa Pecuária Sudeste-Documentos (INFOTECA-E), 2018.

[3] BUENO-LÓPEZ, LILIANA; CAMARGO- GARCIA, JUAN-CARLOS. Nitrógeno edáfico y nodulación de Lecaena leucocephala (Lam.) de Wit en sistemas silvopastoriles. Acta Agronómica, v. 64, n. 4, 2015, p. 349-354. http://dx.doi.org/10.15446/acag.v64n4.45362

[4] ESCALANTE, EDUARDO. Use and performance of leucaena (Leucaena leucocephala) in Venezuelan animal production systems. Tropical Grasslands-Forrajes Tropicales, v. 7, n. 4, 2019, p. 407-409. https://doi.org/10.17138/tgft(7)407-409

[5] KANT, RAVI; BISHIST, ROHIT; KUMAR, MANOL. Effect of Supplementation of Leucaena leucocephala (Lam.) de Wit (Leucaena) Leaves on Growth Profile of Crossbred Calves. International Journal of Livestock Research, v. 9, n. 5, 2019, p. 154-159. 10.5455/ijlr.20181212024424

[6] MATTOS-RIBEIRO, VANDJORE; VALMORBIDA, RAQUEL; DALPIVA-HARTMANN, KATIA-CRISTINA; PORTO, ERLY-CARLOS; ALMEIDA, JULIANA; MALAGUTTI-CORSATO, JAQUELINE; TEIXEIRA-FORTES, ANDRÉA-MARIA. Efeito alelopático de Leucaena leucocephala e Hovenia dulcis sobre germinação de Mimosa bimucronata e Peltophorum dubium. Iheringia. Série Botânica, v. 74, 2019, p. 1-7. https://doi.org/10.21826/2446-82312019v74e2019006

[7] CONRAD, KATHRYN; DALAL, RAM; DALZELL, SCOTT; ALLEN, DIANE; FUJINUMA, RYOSUKE; MENZIES, NEAL. Soil nitrogen status and turnover in subtropical leucaena-grass pastures as quantified by ס15N natural abundance. Geoderma, v. 313, 2018, p.126-134. https://doi.org/10.1016/j.geoderma.2017.10.029

[8] KAMINSKI, P.E.; SCHIFINO-WITTMANN, M.T.; PAIM, N.R. Growth and survival of a range of Leucaena species in southern Brazil. Tropical Grassland, v. 39, 2005, p. 1-8.

[9] RAMÍREZ-BAHENA, MARTHA-HELENA; FLORES-FÉLIX, JOSÉ-DAVID; VELÁZQUEZ, ENCARNA; PEIX, ÁLVARO. The Mimosoid tree Leucaena leucocephala can be nodulated by the symbiovar genistearum of Bradyrhizobium canariense. Systematic and Applied Microbiology, v. 43, n. 1, 2020, p. 126041. https://doi.org/10.1016/j.syapm.2019.126041

[10] KANG, XIA; YU, XIUMEI; ZHANG, YU; CUI, YONGLIANG; TU, WEIGUO; WANG, QIONGYAO; LI, YANMEI; HU, LANFANG; GU, YUNFU; ZHAO, KE; XIANG, QUANJU; CHEN, QIANG; MA, MENGGEN; ZOU, LIKOU; ZHANG, XIAOPING; KANG, JINSAN. Inoculation of Sinorhizobium saheli YH1 Leads to Reduced Metal Uptake for Leucaena leucocephala Grown in Mine Tailings and Metal-Polluted Soils. Frontiers in Microbiology, v. 9, 2018, p. 1-13. 10.3389/fmicb.2018.01853

[11] CUBILOS-HINOJOSA, JUAN-GUILLERMO; MILIAN-MINDIOLA, PABLO-ERNESTO; HERNÁNDEZ-MULFORD, JORGE-LUIS; PERALTA-CASTILLA, ARNALDO. Biological fixation of nitrogen by native isolates of Rhizobium sp. symbionts of Leucaena leucocephala (Lam.) De Wit. Acta Agronómica, v. 68, n. 2, 2019, p. 75-83. 10.15446/acag.v68n2.69322

[12] LEMES-DOS SANTOS, FRANCIANE. Inoculação e coinoculação de rizobactérias promotoras de crescimento em plantas de arroz, milho e trigo [Ph.D Thesis Ciência do Solo]. Porto Alegre (Brasil): Universidade Federal do Rio Grande do Sul, Faculdade de Agronomia, 2018, 106 p.

[13] SARRUGE, J.R. Soluções nutritivas. Summa Phitopathologica, Botucatu, v. 1, n. 3, 1975, p. 231-233.

[14] GOULART-MACHADO, RAFAEL; SACCOL-DE SÁ, ENILSON-LUIZ; OLDRA, SUÉLEN; DALLA-COSTA, MURILO; DALLAGNOL, GILBERTO; DA SILVA-SANTOS, NEEMIAS; ROSA-DA SILVA, WILLIAM. Rhizobia isolation and selection for serradella (Ornithopus micranthus) in Southern Brazil. African Journal of Microbiology Research, v. 10, 2016, p. 1894-1907.

10.5897/AJMR2016.8327

[15] TZEC-GAMBOA, MAGNOLIA; SOLORIO-SÁNCHEZ, FRANCISCO; FIEBRIG, IMMO; TORRES-CALZADA, CLAUDIA; PEÑA-CABRIALES, JUAN J.; ORTIZ-VAZGUEZ, ELIZABETH. Biochemical and Molecular Characterization of Native Rhizobia Nodulating Leucaena leucocephala with Potential Use as Bioinoculants in Yucatan, Mexico. Chiang Mai Journal of Science, v. 47, n. 1, 2020, p.1-15. 
[16] BROCKWELL, J.; HELY, F.W.; NEAL-SMITH, C.A. Some symbiotic characteristics of rhizobia responsible for spontaneous, effective field nodulation of Lotus hispidus. Australian Journal of Experimental Agriculture and Animal Husbandry, v. 6, 1966, p. 365-370.

https://doi.org/10.1071/EA9660365

[17] MENEGHETTI, A.M. Manual de Procedimentos de amostragem e análise química de plantas, solo e fertilizantes. Curitiba (Brasil): Editora da Universidade Tecnológica Federal de Paraná, 2018, 251 p.

[18] EL KADER, ABED; MOLINA, E.A.; MONTERO, K.C.; GUTIERREZ, O.; TRONCONE, G.; LEÓN-DE PINTO, G.. Datos analíticos de la goma de la semilla de Leucaena leucocephala. Revista de la Facultad de Agronomía de La Universidad del Zulia, v. 25, n. 1, 2008, p. 95-118.

[19] RINCÓN, J.; CLAVERO T.; RAZZ, R., PIETROSEMOLI, S., MENDEZ F.; NOGUERA, N. Efecto de la inoculación con cepas nativas e introducidas de Rhizobium sobre la fijación de nitrógeno en Leucaena (Leucaena leucocephala (Lam) de Wit). Revista de la Facultad de Agronomía de la Universidad del Zulia, v. 17, n. 4, 2000, p. 342-357.

[20] HERNÁNDEZ-MULFORD, JORGE-LUIS; CUBILLOS-HINOJOSA, JUAN GUILLERMO; MILIAN, PABLO-ERNESTO. Aislamiento de cepas de Rhizobium sp., asociados a dos leguminosas forrajeras en el Centro Biotecnológico del Caribe. Revista Colombiana de Microbiología Tropical, v. 2, 2012, p. 51-62.

[21] PINTO-RAMOS, DANIELLE-BRAULE; GOMEZ-DE SOUZA, LUIZ-AUGUSTO. Seleção de estirpes de rizóbios para formação de mudas de leucena (Leucaena leucocephala (Lam) De Wit.) em Argissolo Vermelho Amarelo. Revista Brasileira de Agroecologia, v. 8, n. 1, 2013, p. 28-39. 